\title{
Peripheral Zone of Prostate
}

National Cancer Institute

\section{Source}

National Cancer Institute. Peripheral Zone of Prostate. NCI Thesaurus. Code C128570.

The region of the prostate that is located most superficial and posterior as compared to the other prostate zones. It is located closest to the rectum, and is easily palpated on digital rectal exam. This zone of the prostate surrounds the distal urethra, and is the zone of orig in for the vast majority of prostatic cancers. 